\title{
REMARKS PRECEDING A DEMONSTRA- TION RELATING TO THE PRACTICE OF MALARIATHERAPY AT HORTON *
}

\author{
By S. P. JAMES, M.D., F.R.S.
}

THE demonstration which has been prepared for your inspection this afternoon illustrates some details relating to the therapeutic practice of giving patients an attack of malaria with the object of killing the causal organism of syphilis (Spirocheta pallida) when it is deeply embedded in the tissues of the brain as in cases of general paralysis. It is usually admitted that when once this organism has become implanted in the central nervous system it is exceedingly difficult to eradicate it. Malaria, with or without drug treatment, seems to be able to do so in a considerable number of cases, and, as this is so, it is perhaps surprising that the practice of malariatherapy is not extending more rapidly in this country. Up to the present it has been tried in only about half the county and borough public mental hospitals in England and Wales, and the number of patients so treated in any of the last five years has not exceeded 640. This must be regarded as a small number in view of the knowledge that between I,IOO and I,200 deaths from general paralysis occur every year. One would have expected that during the ten years which have elapsed since malariatherapy was officially recommended in this country it would have become the rule not only to utilise the practice for every diagnosed case of general paralysis, but for many syphilitic patients whose spinal fluid showed definite pathological changes, but in whom clinical signs and symptoms of general paralysis were not apparent. The prospect of killing the spirochete in these cases is, of course, much brighter than it is in cases in which the occurrence of mental symptoms proves that the organism has become colonised in the brain tissue. England is

* Delivered before the M.S.S.V.D., at the Annual General Meeting, July I5th, I933. 


\section{PRACTICE OF MALARIATHERAPY AT HORTON}

more backward than France, Germany and some other continental nations in this matter, for as yet the efforts of Colonel Harrison and others to make arrangements for utilising malariatherapy for the prevention as well as for the cure of general paralysis have not materialised. Perhaps you may consider that this is a subject with which your Society should concern itself. One of the reasons why malariatherapy is not more widely used in this country may be that as yet it is not always easy to arrange to give a patient a course of malaria under suitable conditions. From lack of adequate arrangements and facilities the great majority of syphilitic patients who would be most likely to benefit from malariatherapy are debarred from this form of treatment. What is needed is that the L.C.C. or some voluntary body interested in the treatment of venereal diseases should set aside a hospital where syphilitic patients could undergo the treatment, without restriction, before they become mentally deranged. It is a grave misfortune that such a hospital has not yet been established in London.

In addition to this general matter I should like to bring to your notice one or two points with which to-day's demonstration is particularly concerned.

In the first place you will see that at Horton several species and varieties of the malaria parasites are used for inducing the malarial attacks. In the microscope slides you will see the parasite of four different species; and in the wards you will see the types of fever which those species produce. In endeavouring to ascertain how malaria kills the spirochete, and so cures general paralysis, the pyrexial condition itself is one of the first things to study. You will see from these charts that there are great differences between the type of fever produced by the different species of parasite. They show that if the aim is to produce the highest supportable temperature, continued for the longest period that does not involve risk to life, a course of malaria due to the parasite falciparum should clearly be more successful than a course due to the milder forms vivax or ovale or malaria. If the height of the temperature is important one ought to be able to show, by examining a large number of charts, whether the results in cases in which the temperature has exceeded, say, $105^{\circ} \mathrm{F}$. are better than the results 


\section{BRITISH JOURNAL OF VENEREAL DISEASES}

when that temperature has not been reached. If the period of fever is important it is clear that any kind of malaria should give better results than are given by the injection of vaccines or other substances which cause the temperature to rise for only a few hours.

But it is unlikely that temperature is the only factor concerned. Malaria causes profound physical and biochemical changes in the tissues and fluids of the body. A biochemical change which can be studied somewhat easily relates to the composition of the protein constituents of the serum. An attack of malaria causes a marked reduction in the total protein content of the serum from the normal figure of 7.5 or $8 \mathrm{gm}$. per roo c.c. to about $6 \mathrm{gm}$. The reduction is due chiefly to a decrease in the amount of albumin, but there is also some reduction in the amount of total globulin and of pseudoglobulin. The amount of euglobulin is always raised. These changes are not dependent on the actual temperature during the febrile attacks. Many other changes require to be studied if we are to ascertain what factors may be concerned in the action of malaria in killing the spirochete and curing general paralysis.

There is another matter which I think is worthy of systematic study, namely, the effect of specific antisyphilitic drug treatment given while the patient is actually going through the attack of malarial fever. A course of treatment with an arsenical remedy is often prescribed before or after the malaria course, but I have not heard that systematic trials of simultaneous drug treatment and malaria have been conducted. Having in view the difficulty of killing spirochetes which are deeply entrenched in the tissues of the central nervous system, combination of the two plans seems justifiable. On theoretical grounds the drug should be given just before the malarial febrile paroxysm is about to begin in order that both the pyrexia and the drug may act together to the best advantage. Usually this practice cannot be adopted because organic arsenicals, being to some extent antimalarial as well as antisyphilitic remedies, may cut short the malarial attack, but it can be adopted if one uses a species or strain of the malaria parasite against which arsenicals have no action. Such species and strains are available at Horton. In the demonstration you will see all the species of parasites used at 


\section{PRACTICE OF MALARIATHERAPY AT HORTON}

Horton and the method by which they are cultivated in mosquitoes and used for the infection of patients by the bites of these insects. Induction of malaria by mosquito bites is preferable to induction by direct inoculation of blood for the following reasons: (I) It is the only way by which to ensure that the patient is not inoculated with more than one species of the malaria parasite. (2) It is the only way by which to ensure that the patient is not inoculated with some harmful organism such as a streptococcus as well as with the malaria parasite. (3) It is the only way by which to ensure that the patient will have malaria in its natural form, followed by the persistence of the parasites in his system, and occasional relapses which assist in completing the destruction of spirochetes and so in effecting a permanent cure of general paralysis. (4) In infections by mosquito bites specific antisyphilitic drugs can be given during the incubation period of the malarial attack without delaying the onset of that attack. This cannot be done in cases infected by direct blood inoculation because in those cases the malaria might be cured before it had developed clinically. For these reasons we think that the malarial attack should be induced in all cases by mosquito bites. As yet, even in England, mosquitoes cannot be used for all cases, because Horton is the only centre where mosquitoes are prepared for the purpose and, as you will see when you visit the Centre and Laboratory, the arrangements there are only on a small scale. If the hospital which I have mentioned for the treatment of pre-mental syphilitic cases could be established it would become possible to prepare a much larger supply of infected mosquitoes so that a sufficient number would be always available for use wherever they might be required. In that case it might perhaps be preferable, instead of sending the mosquitoes by hand, as is done at present, to put up in a suitable medium and to send by post the infective organisms (the sporozoites) obtained by dissection of the salivary glands of infected insects. This was a plan which we described in I927. It has all the advantages of mosquito-borne malarial infection without the difficulties associated with the use of the insects themselves or the risks associated with blood inoculation. All that the physician would have to do would be to inject the fluid containing the sporozoites into his patient either 


\section{BRITISH JOURNAL OF VENEREAL DISEASES}

intravenously or subcutaneously. Dr. Bruce Mayne, of the United States Public Health Service, who learnt the method at Horton in 1932, has recently reported its successful application in South Carolina and other parts of the United States 